\title{
Neutrino energy quantization in rotating medium
}

\author{
Alexander Grigoriev*, \\ Skobeltsyn Institute of Nuclear Physics, \\ Moscow State University, 119992 Moscow, Russia \\ Alexander Studenikin $\dagger$ \\ Department of Theoretical Physics, \\ Moscow State University, 119992 Moscow, Russia
}

\begin{abstract}
Exact solution of the modified Dirac equation in rotating medium is found in polar coordinates in the limit of vanishing neutrino mass. The solution for the active lefthanded particle exhibit properties similar to those peculiar for the charged particle moving in the presence of a constant magnetic field. Accordingly, the particle in the rotating matter has circle orbits with energy levels, analogous to the Landau levels in magnetic field. The relevant physical realization of the problem is motion of neutrino inside the rotating neutron star. The feature of such motion to form binding states leads to the prediction of the new mechanism for neutrino trapping. The solution found can be used for detailed description of relativistic and nearly massless neutrino dynamics in neutron stars. Results obtained further develop the "method of exact solutions" in application to particle interactions in presence of dense matter.
\end{abstract}

Recently a method for calculation of various processes of elementary particles interaction proceeding in matter has been developed in a series of our papers (see [1] and references therein). The framework of the method is similar to the Furry representation in quantum electrodynamics and implies the use of exact solutions of the wave equations for particles wave functions $[2,3]$ to account for interaction with matter. The "exact solutions method" has been already applied for description of neutrino propagation in different media and electromagnetic fields and also for evaluation of the quantum theory of the spin light of neutrino [2] and spin light of electron [1,3] in matter, the two recently proposed new mechanisms of electromagnetic radiation produced by a neutrino or an electron moving in matter.

The exact solutions of correspondent modified Dirac equations in cases of a neutrino and an electron moving in matter at rest were obtained in [2] and [3] respectively. Then we we

*ax.grigoriev@mail.ru

†'studenik@srd.sinp.msu.ru 
have continued these studies and investigated neutrino behavior in rotating medium with prospects for applications to neutron stars. We have found [3] (see also the second paper of [1]) the exact solution for the neutrino wave function in the case of rotating medium using the Cartesian coordinates. In this short note below we show how the same problem can be solved using the polar coordinates. The obtained below result contributes to the further development of the "exact solutions method" in application to studies of particle interactions in presence of matter. Note that the employment of polar coordinates in consideration of a neutrino motion in the rotating media fits the symmetry of the problem, therefore the correspondent quantum numbers that determines the neutrino quantum state have quite a natural sense.

Let us consider unpolarized matter consisting of neutrons and rotating around the third axes with the angular velocity $\omega$. For definiteness, we consider below the case of an electron antineutrino motion in such a medium. The problem for different other neutrino species can be solved in a similar way.

Our starting point is the modified Dirac equation [2] accounting for the neutrino interaction with matter,

$$
\left\{\gamma_{\mu} p^{\mu}-\frac{1}{2} \gamma_{\mu}\left(1+\gamma_{5}\right) f^{\mu}-m\right\} \Psi(x)=0
$$

where

$$
f^{\mu}=\frac{G_{F}}{\sqrt{2}}\left(1+4 \sin ^{2} \theta_{W}\right) j^{\mu}=\widetilde{G}_{F} j^{\mu}
$$

The matter current is given by

$$
j^{\mu}=(n, n \mathbf{v})
$$

where $n$ is the invariant matter density and $\mathbf{v}$ being the macroscopic matter speed. In our particular case the matter current can be written as $j^{\mu}=n(1,-\omega y, \omega x, 0)$, where $y$ and $x$ are the spatial coordinates in the plane orthogonal to the rotation axis.

Introducing the notation $\gamma=\widetilde{G}_{F} n \omega$ and using polar coordinates we rewrite the equation (1) in components:

$$
\left\{\begin{array}{r}
m \Psi_{1,2}+i e^{\mp i \varphi}\left[\frac{\partial}{\partial r} \mp \frac{i}{r} \frac{\partial}{\partial \varphi} \pm \gamma r\right] \Psi_{4,3}-\left(E+\widetilde{G}_{F} \pm p_{3}\right) \Psi_{3,4}=0 \\
\left(E \mp p_{3}\right) \Psi_{1,2}+i e^{\mp i \varphi}\left[\frac{\partial}{\partial r} \mp \frac{i}{r} \frac{\partial}{\partial \varphi}\right] \Psi_{2,1}-m \Psi_{3,4}=0
\end{array}\right.
$$

where $p_{3}$ is the neutrino momentum operator. The chiral representation of Dirac matrices is used. The form of the equations implies the following operators to be integrals of motion: the energy $E=i \partial_{t}$, the third momentum component $p_{3}=-i \partial_{3}$, and the third component of the total angular momentum (which is the sum of the orbital and spin momenta, $\left.J_{3}=L_{3}+S_{3}=-i \partial_{\varphi}+\Sigma_{3}\right)$. Therefore we write the solution

$$
\Psi(\mathbf{r}, t)=e^{-i E t+i p_{3} z+i(l-1 / 2) \varphi} \psi,
$$


where the function $\psi$ can be written in the following form

$$
\psi=\left(\begin{array}{l}
\psi_{1} e^{-i \varphi} \\
\psi_{2} e^{i \varphi} \\
\psi_{3} e^{-i \varphi} \\
\psi_{4} e^{i \varphi}
\end{array}\right)
$$

Substituting relations (5) and (6) into the system (44) one finds the following set of equations for the functions $\psi_{i}$ :

$$
\left\{\begin{array}{r}
m \psi_{1}-\left(E+p_{3}+\widetilde{G}_{F} n\right) \psi_{3}+i \sqrt{\gamma}\left[\frac{\partial}{\partial r}+\frac{l}{r}+r\right] \psi_{4}=0 \\
m \psi_{2}+i \sqrt{\gamma}\left[\frac{\partial}{\partial r}-\frac{l-1}{r}-r\right] \psi_{3}-\left(E-p_{3}+\widetilde{G}_{F} n\right) \psi_{4}=0 \\
\left(E-p_{3}\right) \psi_{1}+i \sqrt{\gamma}\left[\frac{\partial}{\partial r}+\frac{l}{r}\right] \psi_{2}-m \psi_{3}=0 \\
i \sqrt{\gamma}\left[\frac{\partial}{\partial r}-\frac{l-1}{r}\right] \psi_{1}+\left(E+p_{3}\right) \psi_{2}-m \psi_{4}=0
\end{array}\right.
$$

In the case of relativistic neutrinos (i.e., in the case of vanishing neutrino mass) the fist and the second pairs of the equations decouple from each other and describe, respectively, active left-handed $(L)$ and sterile right-handed $(R)$ neutrino states.

Now let us turn to the first pair of equations in (7). Expressing one wave function component $\left(\psi_{3}\right.$ or $\left.\psi_{4}\right)$ through another and introducing the substitution $\rho=\gamma r^{2}$ we obtain separate equations for the each of the components,

$$
\begin{aligned}
& \left(\rho \frac{\partial^{2}}{\partial \rho^{2}}+\frac{\partial}{\partial \rho}-\frac{(l-1)^{2}}{4 \rho}-\frac{l}{2}-\frac{\rho}{4}+\frac{\left(E+\widetilde{G}_{F} n\right)^{2}-p_{3}^{2}}{4 \gamma}\right) \psi_{3}=0 \\
& \left(\rho \frac{\partial^{2}}{\partial \rho^{2}}+\frac{\partial}{\partial \rho}-\frac{l^{2}}{4 \rho}-\frac{l-1}{2}-\frac{\rho}{4}+\frac{\left(E+\widetilde{G}_{F} n\right)^{2}-p_{3}^{2}}{4 \gamma}\right) \psi_{4}=0 .
\end{aligned}
$$

These equations are almost identical to those corresponding to the case of charged particle motion in the constant homogeneous magnetic field when the problem is considered in the frame of polar coordinates [5]. Their solutions, that posses physical meaning, are expressed via the Laguerre functions $I_{N, s}$ with $N, s=N-l$ and $l$ being the principal, radial and total orbital momentum quantum numbers. The energy-momentum relation therewith is expressed by the formula

$$
E_{N}=\sqrt{p_{3}^{2}+2 N \gamma}+\widetilde{G}_{F} n
$$

Let us consider the second equation of the system (17). Presenting the functions $\psi_{3,4}$ in the form

$$
\psi_{3}=C_{3} I_{N-1, s}(\rho), \quad \psi_{4}=C_{4} I_{N, s}(\rho) .
$$


and taking into account the relation

$$
\begin{aligned}
i \sqrt{\gamma}\left[\frac{\partial}{\partial r}+\frac{l}{r}+r\right] I_{N-1, s}(\rho)= & \\
& i \sqrt{\gamma \rho}\left[2 \frac{\partial}{\partial \rho}+1+\frac{l}{\rho}\right] I_{N, s}(\rho)=i \sqrt{2 N \gamma} I_{N-1, s}(\rho)
\end{aligned}
$$

we obtain relation between coefficients $C_{3}$ and $C_{4}$, that leads to

$$
\frac{C_{3}}{C_{4}}=-i \frac{E-p_{3}+\widetilde{G}_{F}}{\sqrt{2 n \gamma}} .
$$

The remaining non-defined coefficient is determined by the normalization condition for the wave function. Finally for the relativistic active left-handed antineutrino wave function we get

$$
\begin{aligned}
& \Psi_{L}= \\
& \frac{e^{-i E t+i p_{3} z} e^{i(l-1 / 2) \varphi}}{\sqrt{2 \pi L} \sqrt{\left(E+\widetilde{G}_{F} n-p_{3}\right)^{2}+2 N \gamma}}\left(\begin{array}{c}
0 \\
0 \\
\left(E+\widetilde{G}_{F} n-p_{3}\right) I_{N-1, s}(\rho) e^{-i \varphi} \\
i \sqrt{2 N \gamma} I_{N, s}(\rho) e^{i \varphi}
\end{array}\right),
\end{aligned}
$$

where $L$ in the left-hand side is the normalization length.

The solution for the relativistic sterile right-handed neutrino state can be presented in the plane-wave form

$$
\Psi_{R}=\frac{e^{-i p x}}{L^{3 / 2} \sqrt{\left(p_{3}-E\right)^{2}+p_{1}^{2}+p_{2}^{2}}}\left(\begin{array}{c}
0 \\
0 \\
p_{1}-i p_{2} \\
p_{3}-E
\end{array}\right)
$$

with the vacuum energy-momentum relation $E=p$.

As it follows from (9), we conclude that the transversal motion of an active neutrinos and antineutrinos is quantized in moving matter [4] very much like an electron energy is quantized in a constant magnetic field that corresponds to the relativistic form of the Landau energy levels (see, for instance, [5]). Consider again antineutrino. The transversal motion momentum is given by

$$
\tilde{p}_{\perp}=\sqrt{2 \rho N} .
$$

The quantum number $N$ determines also the radius of the antineutrino quasi-classical orbit in matter (it is supposed that $N \gg 1$ and $p_{3}=0$ ),

$$
R=\sqrt{\frac{2 N}{\widetilde{G}_{F} n \omega}} .
$$

From this it follows, for instance, that low energy (but still relativistic) antineutrinos can have bound orbits inside a rotating star. This can lead to the mechanism of low-energy neutrinos trapping inside rotating neutron stars. 


\section{References}

[1] A.Studenikin, J.Phys.A: Math.Gen. 39 (2006) 6769; A.Studenikin, J.Phys.A: Math.Theor. 41 (2008) 164047.

[2] A.Studenikin, A.Ternov, Phys.Lett.B 608 (2005) 107; A.Grigoriev, A.Studenikin, A.Ternov, Phys.Lett.B 622 (2005) 199; A.Grigoriev, A.Studenikin, A.Ternov, Phys.Atom.Nucl. 69 (2006) 1940.

[3] A.Grigoriev, S.Shinkevich, A.Studenikin, A.Ternov, I.Trofimov, Grav. Cosm. 14 (2008) 248; Russ. Phys. J. 50 (2007) 596; hep-ph/0611128.

[4] A.Grigoriev, A.Savochkin, A.Studenikin, Russ.Phys.J.50 (2007) 845.

[5] A.A. Sokolov and I.M. Ternov, Synchrotron radiation (Oxford: Pergamon Press, 1968). 